Questions vives

\section{Questions Vives}

Recherches en éducation

$N^{\circ} 25 \mid 2016$

L'activité des enseignants face au décrochage scolaire : Quelles difficultés et quelles mises en œuvre professionnelles?

\title{
La dynamique du décrochage local des élèves en classe structurée par un faisceau de préoccupations «travail - jeu »
}

The dynamics of local drop-out structured by two concerns "work - play" of students within the class

\section{Olivier Vors}

\section{(2) OpenEdition}

Journals

\section{Édition électronique}

URL : https://journals.openedition.org/questionsvives/1935

DOI : 10.4000/questionsvives.1935

ISSN : $1775-433 X$

Éditeur

Université Aix-Marseille (AMU)

Édition imprimée

Date de publication : 7 juillet 2016

ISBN : 978-2-912643-49-0

ISSN : $1635-4079$

\section{Référence électronique}

Olivier Vors, «La dynamique du décrochage local des élèves en classe structurée par un faisceau de préoccupations « travail - jeu » », Questions Vives [En ligne], N²5 | 2016, mis en ligne le 05 septembre 2016, consulté le 21 septembre 2021. URL : http://journals.openedition.org/questionsvives/1935 ;

DOI : https://doi.org/10.4000/questionsvives.1935

Ce document a été généré automatiquement le 21 septembre 2021.

\section{cc) (†) $९$}

Questions Vives est mis à disposition selon les termes de la licence Creative Commons Attribution -

Pas d'Utilisation Commerciale - Pas de Modification 4.0 International. 


\section{La dynamique du décrochage local des élèves en classe structurée par un faisceau de préoccupations «travail - jeu »}

The dynamics of local drop-out structured by two concerns "work - play" of students within the class

\section{Olivier Vors}

\section{Le décrochage appréhendé comme un processus dynamique et situé au sein de la classe}

1 Chaque année 140000 jeunes quittent le système de formation initiale sans avoir obtenu un diplôme professionnel ou le baccalauréat (MEN, 2015). Le décrochage scolaire questionne l'efficacité du système éducatif, cependant il est difficile à appréhender. Le terme même de décrochage peut varier en fonction des approches, qu'elles soient institutionnelles, sociologique ou psychologique (Biarnès, Redon \& Troncme, 2003). Le terme de décrochage est considéré comme contradictoire et flou selon Guigue (1998): contradictoire, car il peut aussi bien signifier la démobilisation, l'abandon, que la réussite ; flou car il peut désigner à la fois une action précise ou un processus, c'est-à-dire quelque chose sur du long terme. Il parait donc nécessaire de préciser très tôt de quel type de décrochage nous parlons.

2 Nous orienterons donc notre revue de littérature sur : un décrochage considéré comme un processus long, issu d'un parcours personnel et ayant sa propre dynamique.

Diverses études considèrent le décrochage comme un processus de rupture qui se construit au fur et à mesure de la scolarité de l'élève (e.g. Glasman \& Oeuvrard, 2004 ; Millet \& Thin, 2005 ; Thin, 2003). Afin de pouvoir agir sur le décrochage, il est nécessaire 
de comprendre les éléments jalonnant ce processus. Les conflits, les malentendus et les problèmes de sens sont autant de facteurs pouvant expliquer ce processus de décrochage. Souvent le décrochage provient de l'accumulation de divers de ces facteurs, c'est ce qui en fait toute sa complexité. Broccolichi (1997) montre même que les dossiers scolaires des élèves sortis du système sans qualification donnent à voir de grandes difficultés sur le plan des apprentissages bien avant des signes de rupture de la relation pédagogique. Selon cet auteur, les indicateurs de décrochage dépassent le simple absentéisme et questionnent l'activité des élèves en classe.

4 De plus, certaines recherches ont montré que le décrochage était issu d'un parcours personnel (Flavier \& Moussay, 2014 ; Thin, 2003). Cette perspective a permis de compléter les études quantitatives et macroscopiques par l'analyse des trajectoires individuelles permettant de saisir le parcours de chaque élève (Thin, 2003). D'autres recherches ont analysé l'activité des acteurs afin de comprendre les processus à l'œuvre (Flavier \& Moussay, 2014). Ici la parole est donnée aux acteurs afin de saisir leurs motifs d'agir, les résultats montrent par exemple que c'est un déficit de sens qui peut expliquer le désengagement des élèves comme des enseignants.

5 Enfin, pour pouvoir intervenir suffisamment tôt sur le processus de décrochage, il est important de saisir la dynamique de ce processus. Peu d'études font des analyses compréhensives de la dynamique du décrochage des élèves au sein de la classe (Guérin, Pasco, \& Riff, 2008 ; Hosselet \& Vors, 2013). Alors que pour divers auteurs, le décrochage scolaire ne constitue pas une manifestation repérable concernant une population distincte conjuguant un certain nombre de caractéristiques, en rapport avec des conditions familiales et institutionnelles déterminées mais plutôt un processus coconstruit « en situation », notamment dans les interactions entre enseignants et élèves au sein de la classe (Bonnéry, 2003 ; Bruno-Meard, 2015 ; Méard, 2013).

6 Notre étude entre dans cette continuité en étudiant la dynamique du décrochage local des élèves au sein de la classe par l'analyse de leur activité. Nous considérons le décrochage comme un processus, issu d'un parcours personnel et ayant sa propre dynamique trouvant ses origines à l'intérieur de la classe par des comportements non conformes au travail scolaire. L'objet de notre étude est de comprendre la dynamique du décrochage local du travail scolaire au sein de la classe, en analysant l'activité des élèves in situ en cours d'EPS dans des établissements appartenant à la politique d'éducation prioritaire.

\section{Cadre théorique centré sur l'activité en contexte}

7 L'orientation théorique retenue est celle de l'anthropologie cognitive située (Durand, 2001 ; Saury et al., 2013) qui propose une approche compréhensive en accordant le primat à l'intrinsèque de l'acteur. Les fondements philosophiques de cette approche sont ceux de la phénoménologie (Merleau-Ponty, 1942 ; Sartre, 1960) considérant que chaque individu s'engage dans un monde propre qui le caractérise. Ces fondements vont nous permettre d'accéder à l'expérience des élèves et ainsi de comprendre la dynamique de leur engagement en classe.

Dans cette lignée le cadre théorique et méthodologique utilisé est celui du 'cours d'action' (Theureau, 2006) permettant de rendre compte de la dynamique temporelle de l'expérience des élèves. Il est fondé sur deux postulats. D'une part, toute action est considérée comme située dans un contexte social, spatial, temporel et culturel. 
Autrement dit, l'action est couplée avec la situation dans laquelle elle se déploie (Varela, 1989). Cela implique qu'il est nécessaire d'analyser l'action en relation avec le contexte de la classe pour comprendre le décrochage local en tant que processus au sein de la classe. D'autre part, toute action est porteuse de signification. La signification que l'acteur attribue à ses actes se construit dans l'action (Theureau, 2006). Cette signification émerge de la situation et l'acteur n'en a qu'une conscience diffuse. Le chercheur peut accéder à une partie de ses significations appartenant à la conscience pré-réflexive de l'acteur et comprendre ce qui le pousse à agir. Ainsi, l'analyse de l'activité par ce cadre théorique permet d'accéder à la signification que les élèves attribuent à leurs actions. Le cadre théorique du "cours d'action" s'appuie sur différents objets théoriques: le cours d'expérience, le cours d'action, le cours d'information et le cours de vie (Theureau, 2006).

L'objet théorique retenu est ici est le cours d'action car il permet d'articuler la dimension expérientielle du cours d'expérience et la dimension contextuelle de la situation. Le cours d'expérience correspond à la construction de sens par l'acteur de son activité au fur et à mesure que celle-ci se déroule, c'est-à-dire ce qui est montrable, racontable, commentable et mimable par l'acteur à chaque instant. Le cours d'action articule le cours d'expérience à ses déterminants et effets extrinsèques, c'est-à-dire à l'environnement signifiant pour l'acteur. Trois composantes du cours d'action ont été retenues pour rendre compte de la dynamique de l'activité des élèves: ses préoccupations, ses perceptions, et ses connaissances.

\section{Un contexte d'étude considéré comme « difficile »}

Onze élèves de $6^{\mathrm{e}}$ et $5^{\mathrm{e}}$ ont participé à cette étude. La collaboration entre le chercheur et les élèves a été définie par un cadre contractuel visant à créer les conditions d'une confiance mutuelle par: un accord concernant les objectifs de l'étude, un accord sur le droit à l'image et sur la confidentialité des données, une soumission prioritaire des résultats de l'étude aux acteurs, et une liberté d'engagement constante dans l'étude leur offrant la possibilité de ne pas répondre ou d'arrêter la collaboration à tout moment.

11 Notre recherche s'est déroulée dans des établissements ECLAIR (école collège lycée ambition innovation réussite) à la tête de la politique d'éducation prioritaire française. Ces établissements ECLAIR «concentrant le plus de difficultés en matière de climat scolaire et de violence » $(\mathrm{BO}, 2010)$. Sans les stigmatisés de manière déterministe, ces établissements sont statistiquement ceux qui rencontrent le plus de problèmes en termes de décrochage scolaire (Rochex, 2011). De ce point de vue, ce contexte particulier est considéré comme une situation d'étude privilégiée (Grison \& Riff, 2002) du décrochage en classe.

\section{Méthodologie centrée sur l'expérience locale}

\subsection{Recueil des matériaux}

12 Le recueil des matériaux s'est fait par des enregistrements audio-visuels filmant en plan large les 11 élèves participants durant deux leçons d'EPS chacun. Ces enregistrements ont été suivis de séances d'entretien d'autoconfrontation individuel (Theureau, 2006). Au total, 21 entretiens ont été conduit car un des participants n'a pas faire le deuxième entretien. Un protocole d'entretien particulier a été élaboré eu égard la difficulté de 
l'accès à l'expérience avec des élèves (Guérin \& Méard, 2014 ; Moussay \& Flavier, 2014) et de surcroît avec des élèves en difficulté scolaire (Vors, Joing \& Guérin, sous presse) :

- entretiens courts (inférieurs à une heure) ;

- précision systématique du contrat et de la confidentialité des données : « tu peux tout me dire, ce qui se passe ici, le prof n'en saura rien ", «je ne suis pas là pour dire si c'est bien ou pas, je veux juste comprendre" ;

- beaucoup de temps passé avec chacun des élèves participants à l'étude afin de dépasser 'l'étape de défiance' (Guérin, Riff, \& Testevuide, 2004 ; Vors et al., sous presse) ;

- questionnement directif du chercheur ramenant systématiquement aux images «et toi, qu'est que tu fais là, à ce moment-là » afin d'éviter une position d'extériorité ;

- répétition des entretiens avec les mêmes élèves afin que les élèves comprennent ce qui était attendu d'eux (deux entretiens par élève);

- utilisation de l'humour pour les mettre en confiance : rire avec les élèves, provocations « tu te moques de moi, c'était la semaine dernière et tu me dis que tu t'en souviens plus », etc.;

- encouragements explicites lorsque les élèves exprimaient leur expérience : «super, tu vois ça je n'aurais pas pu le savoir sans toi, si tu ne me le dis pas, moi je ne peux pas le deviner, génial!»;

- arrêt de l'entretien face aux résistances des élèves : « tu veux rien me dire, tu ne te souviens de rien, tu préfères que l'on arrête ? ». Un élève n'a d'ailleurs pas souhaité faire le deuxième entretien.

13 Enfin, la validité des propos recueillis a été mise à l'épreuve en référence à trois principes : par la confrontation à des traces objectives de l'activité telles que la vidéo, par la mobilisation de la controverse et par l'utilisation d'un tri conservant uniquement ce qui appartient à l'expérience vécue de l'élève et en éliminant tout discours réflexif ou explicatif. Le traitement des matériaux s'est fait par une démarche itérative visant à mettre à jour les éléments typiques (Vors \& Gal-Petitfaux, 2014a).

\subsection{Traitement des matériaux}

Le traitement des matériaux a permis de reconstruire le «cours d'action » des 11 élèves en portant la focale sur le décrochage local de chacun au sein de la classe. Les indices retenus pour catégoriser ce décrochage local sont les moments où l'engagement des élèves diverge du travail scolaire. C'est-à-dire les moments où la préoccupation des élèves bifurque vers autre chose que le travail scolaire. Pour saisir la dynamique de ces moments de décrochage, toute la temporalité de chaque leçon a été étudiée. Cela a permis l'analyse de la dynamique du décrochage local au sein de la classe incluant le processus de décrochage ponctuel du travail scolaire et le processus de raccrochage au travail.

Le protocole s'est organisé en quatre étapes. Premièrement, la chronologie de l'activité des élèves a été reconstituée dans un tableau à deux volets, en mettant en concordance temporelle les retranscriptions des données de communication, la description des actions filmées et les données d'entretiens. Deuxièmement, la signification de l'activité des élèves a été identifiée à travers leurs préoccupations, perceptions et connaissances afin de repérer les moments de décrochage local. Troisièmement, les processus de décrochage, raccrochage au travail scolaire ont été mis à jour en identifiant les récurrences intra et interindividuelles propres à chacun de ces processus. L'analyse a consisté à comparer et faire résonner entre elles les occurrences de signification et à les configurer sous forme 
de catégories conceptuelles abstraites. Quatrièmement, la dynamique de décrochage local a été analysée en croisant les différents processus à l'œuvre.

Les résultats seront illustrés par différents extraits de cours d'action des élèves participants. Pour alléger le texte, nous ne présenterons pas systématiquement l'ensemble du cours d'action mais seulement des passages focalisant soit sur un extrait d'entretien d'autoconfrontation, soit sur une composante de l'expérience (préoccupation, perception, ou connaissance), soit sur une verbalisation en classe, ou soit sur une description des actions au sein de la leçon. Ces passages choisis dans la présentation des résultats visent à donner un exemple typique afin d'illustrer le propos. Autrement dit, même si l'exemple ne s'appuie que sur un extrait d'étude de cas, il est emblématique de l'analyse intra et inter cas. Il y a donc une différence entre la construction des résultats qui passe par une analyse rigoureuse de l'ensemble des cours d'action et leur présentation qui ne s'appuie que sur quelques passages caractéristiques.

\section{Résultats : la dynamique du décrochage local}

17 Les résultats montrent que le décrochage local des élèves en classe s'appuie sur un faisceau de préoccupations «travail - jeu » comprenant une dynamique propre alternant des phases de décrochage, de raccrochage et d'errance.

\subsection{Activité bipolaire « travail-jeu »}

18 La structure bipolaire de l'activité se manifeste par l'expression d'une grande diversité de comportements et de significations. Pour illustrer cette grande diversité, prenons l'exemple de Soufiane en cours de gymnastique dans l'atelier 'Voler'. Cet atelier est constitué de deux gros tapis de réception, d'un trampoline et d'une fiche explicative des exercices et de leurs critères de réalisation. En dix minutes, les élèves doivent tester différents sauts: saut allumette (avec une position droite du corps), saut groupé, saut carpé, saut demi-tour, saut tour complet. Soufiane alterne entre des actions conformes aux attentes de l'enseignant (faire l'exercice demander, regarder la fiche, attendre son tour pour passer,...) et d'autres ne correspondant pas au travail scolaire demandé (faire des sauts périlleux, discuter, sortir de son atelier, shooter sur le tapis,...). Cet extrait de Soufiane est emblématique de l'activité de tous les élèves étudiés. Il met en évidence une grande diversité de comportements. Les comportements des élèves sont caractérisés par leur courte durée et leur variété.

19 De plus, l'analyse des matériaux d'autoconfrontation montre que les préoccupations qui animent les élèves varient constamment au fil de l'action. Elles sont diverses et de courte durée. Pour revenir à l'exemple précédent de Soufiane dans l'atelier 'Voler' la grande diversité de ses comportements est en lien avec l'instabilité de son engagement. Ses préoccupations bifurquent fréquemment, elles ne durent en moyenne qu'une quinzaine de secondes. Le cours d'action de Soufiane est structuré par la succession des actions suivantes: il cherche à tester le saut demi-tour pour préparer l'évaluation; puis, il s'amuse en se donnant en spectacle devant ses camarades par un salto; il retente le saut demi-tour qu'il vient d'échouer pour vérifier sa difficulté ; puis il s'amuse en perturbant son camarade; il rigole avec le groupe d'élèves de son atelier en faisant un concours de saut «kamikaze »; il prépare l'évaluation, en expérimentant d'abord les sauts qu'il n'a pas encore testés, puis en regardant la fiche, et enfin en appelant le professeur. Au-delà 
de cet exemple particulier, le cours d'action des 11 élèves lors des 21 entretiens d'autoconfrontation est organisé de la même manière avec une grande diversité de comportements et des significations instables.

L'activité des élèves est structurée par un faisceau de deux préoccupations typiques «travailler les exercices prescrits par l'enseignant» et «s'amuser à se provoquer entre pairs ». L'engagement des élèves alternant de façon continue entre le travail et le jeu. Cette forme bipolaire de l'activité est récurrente dans les quatre ateliers et chez tous les élèves étudiés. Les préoccupations "s'amuser» et "travailler " constituent un faisceau toujours présent qui oriente leur action: elles s'actualisent tour à tour en fonction d'éléments de la situation faisant sens pour l'élève. La dynamique d'ouverture et de fermeture de ces deux préoccupations se comprend dans un double processus de décrochage et de raccrochage à la tâche travail scolaire demandée.

\subsection{Processus de décrochage : bifurcation vers la préoccupation de jouer}

21 L'activité des élèves est constamment structurée par une préoccupation de jeu. Même lorsqu'ils travaillent, les élèves sont en quête d'actions ludiques. La préoccupation de jouer est toujours présente, ce qui ouvre des possibles dans l'action des élèves. Le processus de décrochage se caractérise ici par une bifurcation de la préoccupation consistant à "faire le travail demandé » vers celle de "jouer ». Pour comprendre ce processus de décrochage local, nous avons analysé les éléments de la situation provoquant une bifurcation de la préoccupation. Ces décrochages locaux du travail scolaire correspondent à la perception d'éléments qui font signe pour l'élève dans la situation. Ces perceptions typiques fermant la préoccupation de travailler et actualisant la préoccupation de jouer sont en lien avec cinq éléments de la situation : l'ennui de la répétition, la présence de pairs voulant s'amuser, la position éloignée de l'enseignant, les propriétés ludiques du matériel, leur perception négative de la tâche.

Premièrement, les élèves travaillent rarement très longtemps, se lassant vite de la répétition des exercices gymniques : "c'est embêtant de faire toujours la même chose, il faut que ça change " (Extrait d'entretien d'autoconfrontation de Jennifer). Cette phrase de Jennifer est emblématique de ce que ressentent les élèves. La plupart du temps, les élèves perçoivent la répétition comme inutile. En effet, s'ils réussissent dès la première fois, ils jugent qu'ils maîtrisent l'exercice et qu'ils n'ont donc pas besoin de le refaire. Fort de cette réussite ponctuelle, ils estiment qu'ils peuvent alors s'amuser et s'engagent alors dans des actions de jeu : « je me suis dit ça me laisse un peu de temps pour m'amuser » (Extrait d'entretien d'autoconfrontation de Dylan). A contrario, face à l'échec, ils n'essaient pas plus de trois fois consécutives l'exercice en considérant qu'ils ne peuvent pas y arriver. Dans les deux cas, ces jugements ferment souvent la préoccupation de travailler. Ainsi la répétition qu'ils jugent monotone, ennuyeuse, les conduit à passer d'une préoccupation de travail à une préoccupation de jeu.

Deuxièmement, la présence de pairs est une autre source d'actualisation de la préoccupation "s'amuser à se provoquer». Les résultats montrent deux perceptions typiques induisant une bifurcation de préoccupations : la position délicate d'un camarade ou la présence proche d'un camarade voulant s'amuser. La Figure 1 illustre la relation entre la présence de pairs (s'exerçant au même exercice que lui) et les décrochages locaux de Soufiane dans l'atelier « Tourner » comportant quatre exercices différents. Elle met en 
évidence que Soufiane s'entraine à faire la roulade principalement lorsqu'il se trouve seul sur l'exercice.

Figure : Extrait de comportement mettant en relation la présence des pairs de Soufiane avec ses décrochages locaux

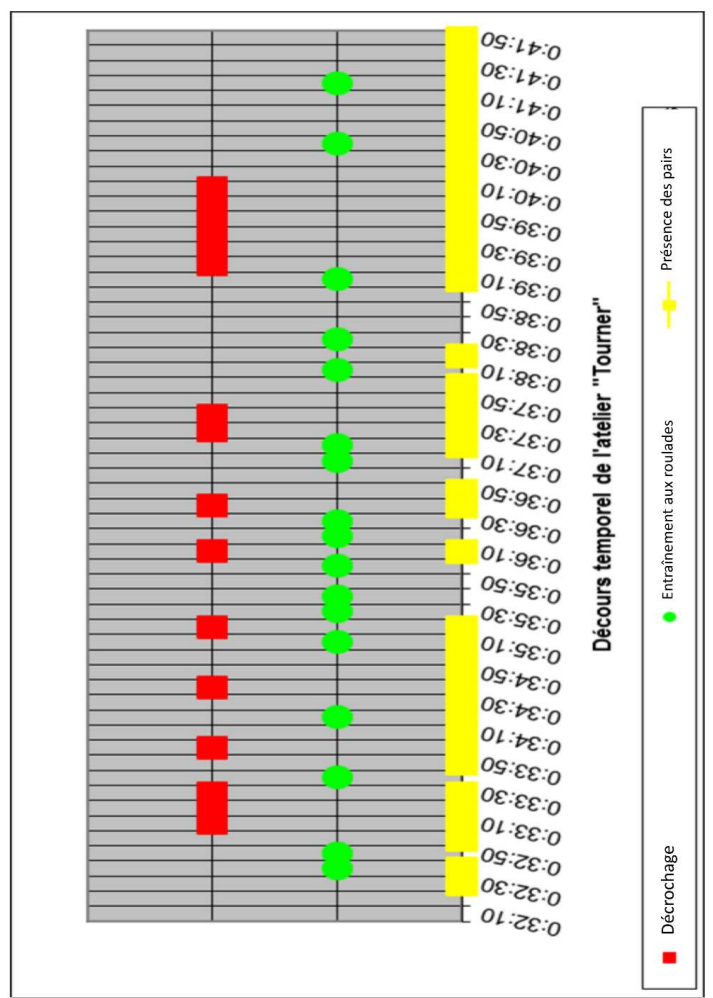

24 La figure 1 met en évidence que la totalité des décrochages locaux (huit au total) du travail scolaire de Soufiane correspond à des moments où ses camarades sont proches de lui. Et inversement, il s'entraine à faire l'exercice demandé majoritairement (10 fois sur 17) lorsque ses pairs ne sont pas dans son environnement proche.

Les matériaux d'entretien d'autoconfrontation permettent de compléter cette description extrinsèque. Ils montrent que la présence des camarades offre à Soufiane des opportunités d'actualiser sa préoccupation de "s'amuser à se provoquer en se faisant des coups en douce ». Après avoir effectué sa roulade, la position de ses camarades fait sens pour lui à quatre reprises (codage en rouge $0: 33: 10,0: 33: 50,0: 34: 30$ et $0: 35: 20$, Figure 1). La posture de ses camarades au départ de la roulade sur le grand plan incliné (position de déséquilibre en contre haut, la tête en bas) est une perception qui ferme sa préoccupation de «s'entrainer à l'exercice le plus difficile » et actualise sa préoccupation de « s'amuser à surprendre son camarade en le faisant tomber ».

De même, les bifurcations de sa préoccupation de travailler se retrouvent lorsqu'un camarade situé à proximité a envie de s'amuser. C'était le cas du premier extrait d'Abdelhamid et Jennifer où la préoccupation de Jennifer a bifurqué en voyant Abdelhamid faire la course. C'est le cas aussi par exemple de Soufiane qui décroche temporellement de la tâche demandée et s'amuse à chahuter avec Aris $(0: 39: 10$, Figure 1):

Extrait de l'activité de Soufiane. Aris vient rejoindre Soufiane et insiste pour faire une roulade à deux, côte à côte, sur le grand plan incliné : «allez, t'as peur ou quoi ». Puis, à la réception des roulades, Aris tombe volontairement sur Soufiane. 
Ceci provoque la réaction immédiate de Soufiane qui lui donne des coups de genou tout en rigolant et en disant " ah aie, aie, tu m'as niqué ». Puis, ils se courent après. par les élèves comme particulièrement propices à « s'amuser à faire des coups en douce » à ses camarades. Le matériel place l'élève qui exécute l'exercice dans une position délicate de déséquilibre et de cécité. Par exemple, l'usage fait par les élèves de l'atelier 'Se renverser' est propice à s'amuser à faire des "coups en douce». Pour réaliser un renversement en appui manuel, l'élève se trouve dans une position délicate, la tête en bas avec un camarade pareur qui doit l'aider en lui tenant les jambes. Dans cette position renversée, l'élève est vulnérable car il ne peut pas se défendre et ne voit pas ce qui se passe derrière lui. Dans cette situation, le camarade pareur actualise souvent sa préoccupation de s'amuser à "taquiner son camarade». Néanmoins, ce type de comportements n'est pas systématique ; le potentiel du matériel offre des ouvertures, des opportunités que les élèves saisissent ou pas. Par exemple, Mohamed et Aris profitent de cette position de faiblesse de Soufiane pour lui retenir les jambes et lui baisser le pantalon.

31

Cinquièmement, le dernier élément de la situation qui fait souvent signe aux élèves lorsqu'ils décrochent est en lien avec leur perception négative de la tâche : la trop grande difficulté ou facilité perçue de l'exercice, la longueur de la file d'attente, l'immobilité trop grande que demande l'atelier, le caractère rapidement fatigant des exercices. Ces différentes caractéristiques de la tâche sont perçues par les élèves comme des opportunités de changement de préoccupation : 
Par exemple après quelques répétitions, Anthony et Ali trouvent l'atelier 'Tourner' trop statique (16 Anthony 2/6). Ils improvisent alors un circuit en enchainant d'un exercice à l'autre au sein du même atelier. Ils prennent de l'élan pour se lancer sur le grand plan incliné; enchaînent une roulade mal maîtrisée; puis, ils courent jusqu'au mur, se repoussent contre celui-ci et s'élance sur le petit plan incliné où ils tentent une roulade arrière sans pouvoir se relever. Après quelques répétitions, le circuit n'est plus considéré comme un prétexte pour effectuer les exercices, le jeu prend le dessus sur le travail. L'exécution des exercices prescrits est bâclée; la préoccupation des élèves se tournant vers un jeu domine ; elle consiste à enchaîner le plus rapidement possible un circuit improvisé. Anthony et Ali prennent dès lors de l'élan pour se jeter à plat ventre sur le grand plan incliné puis réalisent un saut spectaculaire en se servant des qualités de ressort du petit plan incliné. Les élèves expriment « là, on s'amuse à faire n'imp. (n'importe quoi). (...) C'est à celui qui fera le plus le fou, on rigole bien ».

\subsection{Processus de raccrochage : bifurcation vers la préoccupation de travailler}

L'engagement des élèves est toujours constitué par le faisceau de ces deux préoccupations : même lorsque les élèves jouent, la préoccupation de travailler n'est pas totalement fermée. La présence constante des deux préoccupations rend la préoccupation de jouer instable et provoque de nombreuses bifurcations. Les résultats montrent que la fermeture de la préoccupation de jouer et l'actualisation de la préoccupation de travailler sont liées à cinq perceptions typiques en lien avec : les interventions de l'enseignant, la lassitude des jeux, les autres élèves, les caractéristiques de la tâche, le contexte des jeux.

Premièrement, les éléments de bifurcation les plus évoqués en entretien sont liés à l'enseignant: ses interventions, sa présence physique et son influence. Tout d'abord, les interventions de l'enseignant sont souvent des perceptions qui induisent une bifurcation immédiate de la préoccupation de jeu chez les élèves. Ces derniers sont préoccupés à montrer à l'enseignant qu'ils travaillent :

Extrait d'entretien d'autoconfrontation de Romain.

ROMAIN : On jouait à la bagarre, mais après on a arrêté...

CHERCHEUR : Qu'est-ce qui vous a fait arrêter?

ROMAIN : Le prof il nous a pris en flag (en flagrant délit)... il veut qu'on obéisse,

qu'on fasse la consigne!!

CHERCHEUR : Il est embêtant Monsieur W. là ?

ROMAIN : Ben non! C'est un prof, donc il doit le dire.

Toutes les interventions de l'enseignant ne sont pas significatives pour les élèves. Les résultats montrent deux situations typiques où l'intervention de l'enseignant fait signe pour eux et provoque une bifurcation de leur préoccupation de jouer : la compréhension de la remarque de l'enseignant, et le ton qu'il adopte. Dans l'extrait précédent ce qui a fait 
sens pour les élèves n'est pas tant l'intervention de l'enseignant mais le fait qu'ils la trouvent justifiée. De plus, les élèves jugent de l'importance de l'intervention de l'enseignant en fonction de l'énervement qu'ils perçoivent chez l'enseignant :

Extrait d'entretien d'autoconfrontation d'Anthony.

CHERCHEUR : Et là tu le fais bien (l'exercice)?

ANTHONY : Ben ouais!

CHERCHEUR : Non parce que tout à l'heure quand il (l'enseignant) était là, tu lui as montré du catch!

ANTHONY : Non mais là je voyais qu'il commençait à s'énerver un petit peu...

CHERCHEUR : Tu vois ça toi ?

ANTHONY : Il a parlé un peu plus méchamment, il a dit «il faut arrêter ça » tandis que toute à l'heure, il avait dit «il faut arrêter ça » mais il avait parlé plus gentiment.

Ensuite, la simple présence physique de l'enseignant fait s'actualiser la préoccupation de travail des élèves. En voyant l'enseignant s'approcher de leur atelier, les élèves changent de comportement. Ils en profitent souvent pour lui demander un conseil ou la validation de l'exercice "Monsieur, Monsieur regardez si c'est bien" (Verbalisation de Maureen dans l'atelier 'Tourner'). Ce qui fait signe pour certains d'entre eux est le changement rapide de comportement de leur camarade. Ces changements brutaux leur signifient que l'enseignant arrive, et ils provoquent immédiatement la bifurcation de leurs préoccupations de jeu :

Extrait d'entretien d'autoconfrontation d'Anthony. «Moi, je le surveille pas, quand

je vois que les autres commencent à arrêter de faire les fous, ben j'arrête. »

Enfin, l'influence de l'enseignant fait sens pour les élèves les amenant à bifurquer vers une préoccupation liée au travail scolaire demandé. Les observations extrinsèques montrent qu'après une séquence de jeu, les élèves reviennent d'eux-mêmes à des comportements correspondant aux attentes de l'enseignant. L'étude des entretiens d'autoconfrontation permet de comprendre que l'enseignant, même sans être au côté des élèves, influence leurs actions. L'exemple suivant illustre cette idée :

Extrait de l'activité de Fatima. Fatima fait une roue sur l'atelier roulade alors que ce n'était pas un exercice demandé par l'enseignant. En entretien, elle exprime qu'elle fait quelque chose de gymnique « pour pas que le prof dise qu'elle ne travaille pas ».

Deuxièmement, le sentiment de fatigue et le besoin de changement provoquent le raccrochage des élèves au travail. Après une séquence d'activité ludique, ils reviennent d'eux-mêmes à des actions de travail, lassés du jeu. Lors de l'entretien, ils expriment « qu'il faut que ça change » et ils retournent au travail pour changer. Ainsi, la perception provoquant la bifurcation vers une préoccupation de travail est le sentiment de lassitude et de fatigue généré par le jeu.

Troisièmement, les autres élèves constituent des sources potentielles de raccrochage au travail demandé. Les élèves s'observent beaucoup entre eux. La réussite d'un camarade, le fait qu'il aille voir la fiche, qu'il exécute un nouvel exercice sont des éléments significatifs pour l'élève. Les entretiens permettent de mettre à jour que ces perceptions provoquent une bifurcation de ses préoccupations. Il y a aussi un effet de propagation de la mise au travail : le fait que des élèves se remettent au travail produit un effet d'onde sur les élèves aux alentours :

Extrait d'entretien d'autoconfrontation d'Abdelhamid. Après une série de décrochages de la tâche, Abdelhamid retourne au travail « Je recopie sur les autres ce qu'ils font ». 
41 occasions de raccrochage: de nouveaux exercices, un aspect acrobatique, sa facilité perçue ou difficulté perçue, le peu de temps restant, l'évaluation.

Cinquièmement, la perception de l'environnement matériel de travail est source de bifurcation de la préoccupation vers le travail attendu. Les actions de jeu sont imbriquées aux actions de travail, ce leur permet aisément de raccrocher au travail. Les élèves jouent dans l'espace précis de leur atelier et il est rare qu'ils en sortent. Ils ont la préoccupation de «s'amuser en se provoquant» lorsqu'ils attendent leur tour dans la file d'attente, ou après une réception sur le tapis, ou encore lorsqu'ils se replacent au départ de la file. Cet environnement prévu pour le travail offre des opportunités de raccrochage vers des actions studieuses attendues par l'enseignant. Par exemple lorsqu'un élève s'aperçoit que l'atelier se libère, très rapidement il en profite avant que quelqu'un n'arrive, il saisit cette occasion pour s'entraîner. Cette détection d'« espace libre » est très prégnante chez ces élèves ayant tendance à se "faire des coups en douce" lorsqu'ils sont en train de travailler le même exercice.

Cependant, la dynamique de bifurcation des préoccupations n'est pas induite systématiquement par la perception d'un élément isolé. Les bifurcations proviennent le plus souvent d'une convergence de plusieurs éléments :

Extrait de l'activité de Tony. Tony retourne de lui-même au travail après avoir mis au sol Erguez. C'est la coïncidence de trois perceptions qui provoque cette bifurcation de préoccupation : il constate qu'Erguez capitule car il reste sur le dos et il ne tente plus de se défendre; il voit que Soufiane vient de réussir l'exercice difficile; et il s'aperçoit que le plan incliné vient de se libérer. Alors il court vers le plan incliné préoccupé par l'idée de «réussir la roulade avant sur le grand plan incliné sans se taper le dos au sol pour avoir une bonne note ».

Pour conclure, il a été montré que différentes perceptions faisaient bifurquer les préoccupations des élèves du travail vers le jeu, et inversement. Les bifurcations permanentes entre les préoccupations jouer et travailler confèrent une structure cyclique dyadique à l'activité. Cette notion de circularité est importante à saisir pour comprendre l'activité des élèves. La forme de la démonstration a amené à présenter séparément ces deux préoccupations mais elles sont toujours présentes dans l'action. Dans certains cas, elles s'actualisent toutes les deux en même temps. C'est l'exemple de l'introduction où Abdelkarim et Jennifer font l'exercice demandé tout en s'amusant à faire la course. La présence concomitante des préoccupations de jouer et de travailler se retrouve dans de nombreux extraits :

Extrait d'entretien d'autoconfrontation d'Anthony : Le jeu c'était de rigoler un petit peu et de faire les exercices.

Extrait d'entretien d'autoconfrontation de Romain : Je joue avec Omar et en même temps je travaille.

Extrait d'entretien d'autoconfrontation de Tony : On faisait les exercices en s'amusant

Le faisceau dyadique de préoccupations peut s'actualiser de manière très rapide comme cela vient d'être vu. La perception d'un élément particulièrement significatif pour l'élève dans son environnement entraine un changement brutal de préoccupation. Néanmoins, les résultats montrent aussi la présence de phases transitoires dans lesquelles les élèves vagabondent en quête d'occupations. 


\subsection{Processus transitoire : les actions d'errance attentiste} transitoire oscillant entre un engagement tourné vers le travail et un engagement tourné vers le jeu. La phase d'errance attentiste est une période où l'élève vagabonde dans l'atelier en quête d'occupations. L'élève erre aux aguets, prêt à saisir la moindre occasion de changement.

Ces comportements correspondent au moment où les élèves se laissent doubler dans la file d'attente, se couchent sur les tapis, prennent volontairement beaucoup de temps pour se replacer, restent à la parade, vont regarder longuement la fiche, déambulent dans le gymnase. Ils se caractérisent par un rythme lent de leurs déplacements et une observation attentive à ce qui se passe autour d'eux. En situation d'autoconfrontation, les élèves expriment à la fois un sentiment d'ennui "j'en ai marre », « je suis fatigué », " y'a rien à faire » et parallèlement une volonté de changement "il faut que ça change », "je cherche quelque chose à faire", "je regarde ce que font les autres", "je cherche à passer le temps». Ils errent dans l'atelier en cherchant quelque chose à faire. C'est une errance attentiste, car ils sont à l'affut, ils sont en quête d'occasion de changement : ils restent très attentifs à ce qui se passe autour d'eux et en particulier à ce que font leurs pairs.

Cette phase d'enquête est transitoire. Elle s'enchaîne souvent par l'actualisation de la préoccupation de s'amuser ou de travailler. Elle fait partie intégrante de la dynamique de l'activité bipolaire des élèves qui est constamment tenue par l'alternance des préoccupations de s'amuser et de travailler.

\section{Discussion : du décrochage local au décrochage scolaire}

Ces études de cas en situation ordinaire en classe, appartenant à des établissements ECLAIR à la tête de l'éducation prioritaire, permet de discuter de la dynamique de décrochage local des élèves au sein de la leçon. Nous avons vu que cette dynamique reposait sur un faisceau de deux préoccupations typiques «travailler les exercices prescrits par l'enseignant » et "s'amuser à se provoquer entre pairs ». Ce faisceau était toujours présent et structurait l'activité des élèves de manière bipolaire, l'engagement des élèves alternant de façon continue entre le travail et le jeu. La dynamique d'ouverture et de fermeture de ces deux préoccupations se comprend dans un double processus de décrochage et de raccrochage au travail scolaire demandé. Cette dynamique de décrochage local semble avoir une spécificité particulière aux milieux considérés comme «difficiles» par l'institution. Ces décrochages locaux et situés sont caractéristiques par leur nombre et leur furtivité. La fréquence d'apparition des décrochages du travail scolaire dans la classe est quasi permanente. Leur nombre est bien supérieur aux résultats précédemment constatés dans des établissements n'appartenant pas aux politiques compensatoires, mentionnant un comportement déviant de la tâche prescrite toutes les trois ou quatre minutes (Piéron \& Emonts, 1988) ou encore un incident toutes les deux minutes (Turcotte et al., 2008).

De plus, la dynamique de ces décrochages locaux que nous avons observée est originale par sa vitesse de propagation. Les décrochages du travail scolaire ont une forte dimension 
sociale étant liés à la préoccupation de "s'amuser à se provoquer entre pairs». Leur dynamique est très rapide, nous avons montré que pour pouvoir jouer, un élève invite un autre élève à décrocher du travail scolaire, puis un autre élève qui les voit se joint à eux, etc. Cette propagation rapide dans la classe peut vite dégénérer en provoquant le décrochage de la tâche de la plupart des élèves de la classe. Cette particularité conforte la thèse de «l'effet loupe » de l'éducation prioritaire (Kherroubi \& Rochex, 2004); ce qui se passe dans ces classes appartenant à l'éducation prioritaire met à jour des phénomènes également présents, mais moins repérables, dans les établissements et quartiers « ordinaires ». Les décrochages locaux du travail scolaire existent dans toutes les classes, mais sont plus fréquents et ont une dynamique plus rapide dans les classes ECLAIR.

Pour généraliser nos propos, discutons des relations entre ces décrochages locaux au sein de la classe et le décrochage scolaire total menant à la déscolarisation (Glasman \& Oeuvrard, 2004). La dynamique de décrochage local que nous avons mise à jour est-elle un précurseur du décrochage total?

Les processus du décrochage local que nous avons mis en évidence se différencient de ceux propre au " décrochage de l'intérieur » décrit par Bonnéry (2003) ou Broccolichi et Ben-Ayed (1999). Pour ces auteurs, le décrochage de l'intérieur se manifeste lorsque l'élève renonce aux apprentissages en classe pensant de plus en plus souvent que le jeu scolaire n'est plus jouable. Ce type de décrochage est considéré comme une désaffiliation constituant un indicateur précurseur décrochage total (Biarnès et al., 2003). Dans notre étude, le décrochage local du travail scolaire au sein de la classe suit une autre logique qui n'éloigne pas les élèves du travail et des apprentissages. Même s'ils sont fréquents, ces décrochages furtifs de la tâche prescrite ne peuvent pas être considérés comme propédeutique à un décrochage total. Nos résultats ont montré une dynamique cyclique entre des processus de décrochage et de raccrochage au travail scolaire. Le nombre de décrochage local furtif ne serait donc constituer un bon indicateur du décrochage total. Cette vision rejoint certaines études considérant que les décrochages ludiques dissimulés sont complémentaires au travail en classe (Guérin et al., 2004). D'autres études vont même plus loin en montrant que ces décrochages sont nécessaires pour permettre aux élèves de rester engagés dans la classe (Allen, 1986; Gleyse \& Canal, 2013). Ces décrochages ludiques et furtifs rendent viable l'engagement de l'élève au travail ; ces jeux clandestins apparaissent comme un exutoire permettant au système de se pérenniser. L'engagement au travail constitue donc la différence majeure entre le « décrochage de l'intérieur " associé à un renoncement au travail et une désaffiliation du jeu scolaire (Biarnès et al., 2003; Bonnéry, 2003) et le décrochage local, furtif, mis en évidence dans cette étude, associé à une dynamique de raccrochage au travail scolaire. Cette filiation entre le travail scolaire et le décrochage total se retrouve dans l'analyse des dossiers scolaires des élèves sortis du système scolaire sans qualification qui mettait en évidence la présence de problèmes d'apprentissages bien avant les problèmes pédagogiques (Broccolichi, 1997). D’après nos résultats, ce n'est pas le nombre de décrochage local mais leur durée qui semble être un indicateur plus fiable pour caractériser le désengagement des élèves au travail. Différentes recherches sur l'activité des enseignants et des élèves en attestent (Vors, 2011; Vors \& Gal-Petitfaux, 2014b), en montrant que lorsque les décrochage durent dans le temps, la situation de classe dégénère et les élèves ne retournent plus au travail.

Professionnellement, il est difficile aux enseignants de faire la différence entre un décrochage relevant d'une désaffiliation propre au décrochage de l'intérieur (Biarnès et 
al., 2003; Bonnéry, 2003; Broccolichi \& Ben-Ayed, 1999) et un décrochage local complémentaire au travail scolaire. Or, cette différenciation entre ces deux formes de décrochage semble importante pour que l'enseignant puisse adapter son intervention afin de limiter le décrochage total. Pour saisir le sens du décrochage scolaire des élèves au sein de la leçon, l'enseignant doit tenter de comprendre leur expérience vécue en classe. Cette attitude compréhensive de l'enseignant pourrait éviter les malentendus et les déficits de sens souvent à l'origine du processus de désaffiliation progressive des élèves (Flavier \& Moussay, 2014 ; Méard, 2013 ; Millet \& Thin, 2005) notamment lors de l'entrée dans le secondaire (Bruno-Méard, 2015). Une attitude empathique de l'enseignant permettrait d'intervenir plus efficacement face aux décrochages du travail scolaire des élèves (Yilmaz \& Sahinkaya, 2010). Par empathie, l'enseignant essaierait de se mettre à la place de l'élève et tenterait de comprendre ce qu'il vit (Rogers, 1975). Le fait de comprendre ce qui mobilise l'élève permettrait à l'enseignant d'éviter les frustrations et les incompréhensions qui sont souvent au centre du processus de décrochage (e.g., Glasman \& Oeuvrard, 2004; Thin, 2003). En relation avec nos résultats, une attitude empathique ouvrirait l'accès aux préoccupations des élèves et permettrait à l'enseignant de se focaliser et d'attirer ostensiblement l'attention des élèves sur les raccrochages et sur le travail effectué afin de créer un climat propice aux apprentissages (Vors, GalPetitfaux, \& Potdevin, 2015). La focalisation sur les apprentissages pourrait constituer un levier important de raccrochage permettant aux élèves de donner du sens à leur engagement scolaire (Rochex, 1995).

\section{Conclusion} sein de la classe; même si la généralisation de ces études de cas est toujours à questionner dans d'autres contextes. De plus, l'originalité de cette recherche réside dans la prise en compte du décrochage comme un processus dynamique pendant la leçon. Cette approche qualitative et dynamique permet de donner un autre éclairage heuristique à la compréhension du phénomène complexe du décrochage.

L'analyse de la dynamique du décrochage local pourrait être poursuivie à des empans temporels plus grands que celui de la leçon. Comme le dit Lemke (2000) chaque échelle temporelle apporte son propre niveau d'intelligibilité. La perspective de cette recherche pourrait être d'étudier le parcours personnel d'élèves à l'échelle de leur scolarité. Cette temporalité consisterait à analyser à un grain fin les trajectoires des élèves et permettrait de comprendre (voir de typifier) les bifurcations de ces trajectoires vers un décrochage total. Cela viendrait compléter les travaux déjà engagés sur les parcours personnels des élèves (e.g., Thin, 2003) par une analyse de l'activité des élèves avec l'objet théorique du « cours de vie » (Theureau, 2006). 


\section{BIBLIOGRAPHIE}

Allen, J. D. (1986). Classroom Management: Students' Perspectives, Goals, and Strategies. American Educational Research Journal, 23(3), 437-459.

Biarnès, J., Redon, C., \& Troncme, C. (2003). Du décrochage au raccrochage, ou d'un lien en creux à un lien en plein : Entre sub-cultures différentes et temps différents : Prévenir les ruptures scolaires. VEI Enjeux, 132, 204-225.

Bonnéry, S. (2003). Le décrochage scolaire de l'intérieur : interaction de processus sociaux, cognitifs, subjectifs et langagiers. Les Sciences de L'éducation Pour L'ère Nouvelle, 36(1), 39-57.

Broccolichi, S. (1997). Les abandons d'études avant la fin d'un cycle. Bulletin D'information ONISEP, (518), 3-28.

Broccolichi, S., \& Ben-Ayed, C. (1999). L'institution scolaire et la réussite de tous aujourd'hui : " pourrait mieux faire. » Revue française de pédagogie, 129(1), 39-51. http://doi.org/10.3406/ $\underline{\text { rfp.1999.1063 }}$

Bruno-Meard, F. (2015). Analyse du développement du pouvoir d'agir d'enseignants confrontés au risque de décrochage scolaire :étude de cas en classe de sixième de collège. (Thèse de doctorat STAPS non publiée). Aix-Marseille Université, Marseille. Retrieved from http://www.theses.fr/s109187

Circulaire n 2010-096. (2010). Programme "Clair." Paris: MEN. Retrieved from http:// www.education.gouv.fr/cid52643/mene1017616c.html

Durand, M. (2001). Chronomètre et survêtement. Reflets de l'expérience quotidienne d'enseignants en éducation physique. Paris : Éditions Revue EPS.

Flavier, E., \& Moussay, S. (Eds.). (2014). Répondre au décrochage scolaire. Expériences de terrain. Louvain la Neuve : De Boeck. Retrieved from https://hal.archives-ouvertes.fr/hal-01075014/

Glasman, D., \& Oeuvrard, F. (2004). La déscolarisation. Paris : La Dispute.

Gleyse, J., \& Canal, J. L. (2013). Everyday physical education for 11-year-olds in a "rurban" (outer city) secondary school in the south of France: the conflict between norms and "arts de faire" - an ethnographic approach. Physical Education \& Sport Pedagogy, 18(3), 298-316. http:// doi.org/10.1080/17408989.2012.690373

Grison, B., \& Riff, J. (2002). Validité écologique et situations d'étude privilégiées : de la psychologie expérimentale à l'anthropologie cognitive située. In Objets théoriques, objets de conception, objets d'analyses et situations d'éude privilégiées. Sologne : ACT'ING.

Guérin, J., \& Méard, J. (2014). Conduite de l'entretien auprès des jeunes scolaires : le cas de l'autoconfrontation dans une approche " orientée - activité ». Canadian Journal of Education/ Revue canadienne de l'éducation, 37(1), 120.

Guérin, J., Pasco, D., \& Riff, J. (2008). Activités dissimulée et publique d'un élève décrocheur en mathématiques. Revue Des Sciences de L'éducation Pour L'ère Nouvelle, 41(1), 63-82.

Guérin, J., Riff, J., \& Testevuide, S. (2004). Étude de l'activité « située » de collégiens en cours d'EPS : une opportunité pour examiner les conditions de validité des entretiens d'autoconfrontation. Revue Française de Pédagogie, 147(1), 15-26. 
Guigue, M. (1998). Peut-on définir le décrochage. In M. C. Bloch \& B. Gerde (Eds.), Les lycéens décrocheurs, de l'impasse aux chemins de traverse (pp. 25-38). Lyon : Chronique Sociale.

Hosselet, M., \& Vors, O. (2013). Dynamique de l'activité en classe pour des élèves décrocheurs de l'intérieur. Revue EPS, (355), 42-45.

Kherroubi, M., \& Rochex, J.-Y. (2004). La recherche en éducation et les ZEP en France. 2. Apprentissages et exercice professionnel en ZEP : résultats, analyses, interprétations. Revue Française de Pédagogie, 146, 115-190.

Lemke, J. L. (2000). Across the scales of time: Artifacts, activities, and meanings in ecosocial systems. Mind, Culture and Activity, 7(4), 273-290.

Méard, J. (2013). « L'activité » des élèves en risque de décrochage scolaire en EPS : la dynamique des interactions en classe. eJRIEPS, 30, 99-104.

MEN. (2015). Le décrochage scolaire. Retrieved April 29, 2016, from http://www.gouvernement.fr/ action/le-decrochage-scolaire

Merleau-Ponty, M. (1942). La structure du comportement. Paris: PUF.

Millet, M., \& Thin, D. (2005). Ruptures scolaires :l'école à l'épreuve de la question sociale. Paris : Presses Universitaires de France.

Moussay, S., \& Flavier, É. (2014). L'entretien d'autoconfrontation : la prise en compte du point de vue de l'élève pour développer l'activité en classe. Canadian Journal of Education/Revue Canadienne de L'éducation, 37(1), 96.

Piéron, M., \& Emonts, M. (1988). Analyse des problèmes de discipline dans les classes d'éducation physique. Revue de L'éducation Physique, 28(1), 33-40.

Rochex, J.-Y. (1995). Le sens de l'expérience scolaire : entre activité et subjectivité. Paris : PUF.

Rochex, J.-Y. (Ed.). (2011). La politique ZEP en France, laboratoire des politiques d'éducation? Revue Française de Pédagogie, (177). Retrieved from http://www.cairn.info/revue-francaise-depedagogie-2011-4-page-5.htm

Rogers, C. R. (1975). Empathic: An unappreciated way of being. The Counseling Psychologist, 5(2), $2-$ 10.

Sartre, J. P. (1960). Critique de la raison dialectique. Paris : Gallimard.

Saury, J., Adé, D., Gal-Petitfaux, N., Huet, B., Sève, C., \& Trohel, J. (2013). Actions, significations et apprentissages en EPS : une approche centrée sur les cours d'expérience des élèves et des enseignants. Paris : Éditions EP\&S.

Theureau, J. (2006). Le cours d'action. Méthode développée. Toulouse : Octarès.

Thin, D. (2003). « Déscolarisation » des collégiens de milieux populaires : parcours et configuration. In D. Glasman (Ed.), Programme interministériel de recherches sur le processus de déscolarisation (pp. 59-68). Paris : MEN-DEP.

Turcotte, S., Desbiens, J.-F., Spallanzani, C., Roy, M., Brunelle, J. P., \& Tourigny, J. S. (2008). Portrait des comportements perturbateurs adoptés par des élèves du niveau primaire en éducation physique et à la santé. eJRIEPS, 13, 57-77.

Varela, F. (1989). Autonomie et connaissance. Essai sur le Vivant. Paris : Seuil.

Vors, O. (2011). L'activité collective en classe d'éducation physique dans les collèges ÉCLAIR. Étude anthropologique des situations de travail par ateliers en gymnastique et contribution à la connaissance des 
interactions dans les milieux éducatifs "difficiles" (Thèse de doctorat STAPS non publiée). Université de Clermont-Ferrand 2, Clermont-Ferrand.

Vors, O., \& Gal-Petitfaux, N. (2014a). Formes d'expériences sensorielles structurant les stratégies d'intervention en classe difficile. Recherches et Éducations, 12, 27-44.

Vors, O., \& Gal-Petitfaux, N. (2014b). Relation between students' involvement and teacher management strategies in French "difficult"classrooms. Physical Education and Sport Pedagogy, (ahead-of-print), 1-23.

Vors, O., Gal-Petitfaux, N., \& Potdevin, F. (2015). A successful form of trade-off in compensatory policy classrooms. European Physical Education Review, 21(3), 340-361. http:// doi.org/10.1177/1356336X15569373

Vors, O., Joing, I., \& Guérin, J. (in press). Du corps vécu au corps décrit chez des élèves en difficulté scolaire. Proposition méthodologique d'une co-consctruction de l'accès à leur expérience lors d'entretien d'autoconfrontation. In N. Burel \& B. Andrieu (Eds.), Corps \& méthodologies. Paris : L'Harmattan.

Yilmaz, S., \& Sahinkaya, N. (2010). The relationship between the methods teachers use against the misbehaviour performed in the classroom and emphatic tendencies of teachers. Procedia Social and Behavioral Sciences, 2(2), 2932-2936.

\section{RÉSUMÉS}

Cette recherche considère le décrochage comme un processus, issu d'un parcours personnel et ayant sa propre dynamique trouvant ses origines à l'intérieur de la classe par des comportements non conformes au travail scolaire. L'objet de notre étude est de comprendre la dynamique du décrochage local, situé, en analysant l'activité des élèves en cours d'éducation physique et sportive (EPS) dans des établissements appartenant à la politique d'éducation prioritaire. Le cadre théorique et méthodologique mobilisé est celui du 'cours d'action' (Theureau, 2006). Le décrochage local du travail scolaire est analysé au regard de l'engagement des élèves dans la leçon en fonction de leurs bifurcations de préoccupations. Onze élèves de $6^{\text {ème }}$ et $5^{\text {ème }}$ ont participé à cette étude dans un dispositif de gymnastique par ateliers. Le recueil de matériaux s'est fait à partir d'enregistrements audiovisuels et d'entretiens d'autoconfrontation. Leur traitement a visé à rendre compte de l'activité des élèves et en particulier leurs préoccupations, perceptions et connaissances mobilisées dans l'action. Les résultats montrent que l'activité des élèves est organisée par un faisceau de préoccupations "travail - jeu» comprenant une dynamique propre alternant des phases de décrochage et de raccrochage au travail scolaire.

This research considers the dropout as a process, from a personal course and having its own dynamics finding its origins within the classroom by misbehavior. The purpose of our study is to understand the dynamics of local drop-out by analyzing the activity of students during physical education class in middle schools belonging to the compensatory policy. The theoretical and methodological framework used is the "course of action" (Theureau, 2006). The local dropout of school work is analyzed in terms of student engagement in the lesson according to their concerns bifurcations. Eleven students from $6^{\text {th }}$ and $5^{\text {th }}$ in middle schools were studied in a gymnastics workshops. The collection of data is made from video recordings and interviews of self-confrontation. Data processing aimed to analyze the activity of the students and in particular their concerns, perceptions and knowledge used in action. The results show that the activity of the students is organized by two concerns "work - play" including its own dynamic alternating phases of drop-out and of hang up the school work. 
INDEX

Keywords : décrochage local, activité, dynamique, processus, classe

\section{AUTEUR}

\section{OLIVIER VORS}

Maître de conférences, École supérieure du professorat et de l'éducation (ESPE) Académie d'Aix Marseille, UMR CNRS 7287 ISM 